\title{
TNS1-ALK Fusion in a Recurrent, Metastatic Uterine Mesenchymal Tumor Originally Diagnosed as Leiomyosarcoma
}

\begin{abstract}
Jessica Lee ${ }^{1}$, Arun Singh ${ }^{2}$, Siraj M. Ali ${ }^{1}$, Douglas I. Lin ${ }^{3}$, Samuel J. Klempner ${ }^{4,5}$
${ }^{1}$ Foundation Medicine, Cambridge, MA, USA, ${ }^{2}$ Department of Medical Oncology, University of California Los Angeles, Los Angeles, CA, USA, ${ }^{3}$ Beth Israel Deaconess Medical Center, Boston, MA, USA, ${ }^{4}$ The Angeles Clinic and Research Institute, Los Angeles, CA, USA, ${ }^{5}$ Samuel Oschin Comprehensive Cancer Institute, CedarsSinai Medical Center, Los Angeles, CA, USA

Correspondence:

sklempner@theangelesclinic.org

Tel.: + 13102312167

Fax.: + 13102312172

Objective. We report a female patient diagnosed with a leiomyosarcoma and who harbored a druggable target as identified by comprehensive genomic profiling in the course of clinical care. Case Report. The patient progressed five years after curative intent surgery and adjuvant treatment. After failure of multiple lines of chemotherapy,she was enrolled in a trial of an ALK inhibitor based on comprehensive genomic profiling (CGP) identifying an TNS1-ALK fusion. Conclusion. In this case, identification of the ALK kinase fusion permitted enrollment in a matched mechanism driven clinical trial after exhausting standard of care treatment options. CGP raises the possibility of uterine inflammatory myofibroblastic tumor as an alternative diagnosisto leiomyosarcoma, highlighting the complementary role of CGP beyond immunohistochemical analyses.
\end{abstract}

Received: 16 September 2018

Accepted: 30 April 2019

Key Words: Alk - Leiomyosarcoma

- IMT - Kinase Fusion - CGP.

\section{Introduction}

Leiomyosarcoma (LMS) is a malignant tumor arising from smooth muscle, typically of uterine origin (1). Management of disseminated or recurrent disease is challenging, with cytotoxic chemotherapy being the backbone of treatment despite poor outcomes with a median survival of 27 months from first disease recurrence (2). Diagnosis of LMS, especially of the myxoid type, may also be challenging since a subset of cases may be re-classified as either inflammatory myofibroblastic tumor (IMT) or high-grade endometrial stromal sarcoma (ESS) based on both morphologic, immunohistochemical and molecular features (3). Distinction of these entities may affect therapeutic decision-making as IMT and ESS may be less aggressive and more amenable to targeted therapies than LMS. Specifically, IMTs frequently harbor fusions of the ALK kinase that may be susceptible to ALK inhibitors, well established and approved agents in nonsmall cell lung cancer (4-6). A complementary role for molecular testing in advanced tumors is established, and comprehensive genomic profiling (CGP) has demonstrated ability to both re-classify tumors and distinguish recurrence from a second primary (7).

Here we present a case of a malignant and high stage uterine mesenchymal tumor, originally diagnosed as LMS, which after progression on LMS standard therapy was 
found to harbor an ALK gene fusion by genomic profiling. This molecular finding enabled the patient to be enrolled in a clinical trial with the ALK-inhibitor crizotinib and suggested that the tumor was potentially a previously under-recognized IMT.

\section{Methods}

Comprehensive genomic profiling was performed on the Foundation One Heme panel (Foundation Medicine, Cambridge, MA, USA) in a CLIA certified laboratory. This method consists of the analysis of the coding DNA sequences of 405 cancer-related genes, selected introns of 31 genes involved in chromosomal rearrangements, and the RNA sequences of 265 genes commonly involved in fusions. The genes were sequenced to a median coverage of $859 \mathrm{X}$ and the sequences were analyzed for base substitutions, indels, copy number alterations, and rearrangements.

\section{Case Presentation}

A 39-year old African-American woman initially presented with a diagnosis of leiomyoma and ovarian cyst though concern for invasive disease remained. Therefore, a TAH-LSO was performed, and microscopic examination of the specimen identified a leiomyosarcoma. A completion BSO, omentectomy and tumor debulking was then performed, and LMS involving the omentum, right fallopian, and multiple colonic nodules was confirmed based on immunohistochemical and morphologic examination. She received six cycles of adjuvant gemcitabine and paclitaxel followed by radiation therapy. She was under surveillance with bi-annual imaging when a pelvic/bowel recurrence was identified 4 years from her original diagnosis. After surveillance imaging suspicious for recurrent disease, she underwent a PET-CT notable for a left pel- vic mass superior to the bladder measuring $3.9 \mathrm{~cm}$ with an SUV 10 and a cul de sac mass measuring $4.2 \mathrm{~cm}$ with an SUV of 5.3. She underwent a salvage partial colectomy and pathologic examination identified distal sigmoid colon and proximal rectal masses that were morphologically consistent with metastatic leiomyosarcoma. She was treated with palliative doxorubicin and olaratumab but imaging after the second cycle showed progression with an enlarging pelvis mass measures $5.8 \times 5.6 \mathrm{~cm}$ with SUV 7.3, previously $2.9 \times 3.1 \mathrm{~cm}$ with SUV 6.7. Two months after the third cycle of treatment, a pelvic mass was resected, which was again diagnosed as metastatic LMS and adherent to the small bowel serosa, without bowel wall involvement. A nodule on the sigmoid colon was also interpreted as LMS. Two months after surgery, adjuvant pazopanib at $400 \mathrm{mgs}$ daily was initiated, and then switched to $400 / 600$ $\mathrm{mg}$ alternating daily due to poor tolerance and fatigue.

After 12 months on pazopanib she presented to an emergency room with pain and was found to have progressive disease. She was transitioned to trabectedin but tolerated it poorly, and imaging after two cycles confirmed further disease progression. In an effort to explore all possible treatment options a specimen from the salvage colectomy was sent for comprehensive genomic profiling as previously described (8). CGP identified a predicted oncogenic TNS1-ALK fusion which was also observed in a complementary circulating tumor DNA assay (Figure 1). Based on her CGP results she was screened for a precision medicine clinical trial (NCT02693535). Owing to impaired renal function from extrinsic compression by the large pelvic mass (Figure 1A) she was deemed ineligible. In the absence of ability to get on trial she was transitioned to Brigatinib off-label. Within 2 weeks she had significant improvement in pain and was able to come off all opioids. First imaging re- 

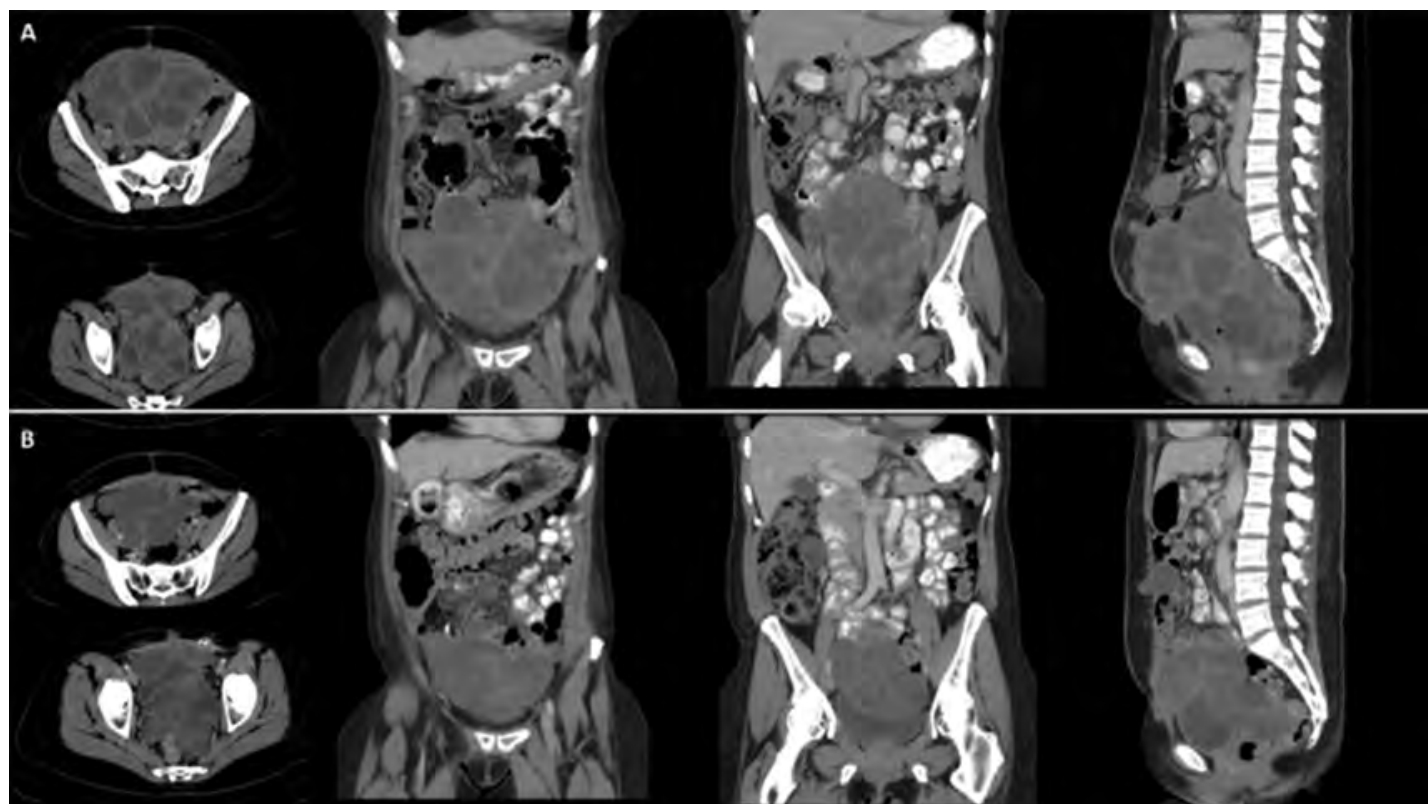

Figure 1. Rapid treatment response in a symptomatic LMS patient whose tumor was found to have an ALK-TNS1 fusion on comprehensive genomic profiling. Images are shown: (A) prior to brigatinib and (B) after only 35 days on brigatinib $180 \mathrm{mg}$ by mouth daily.

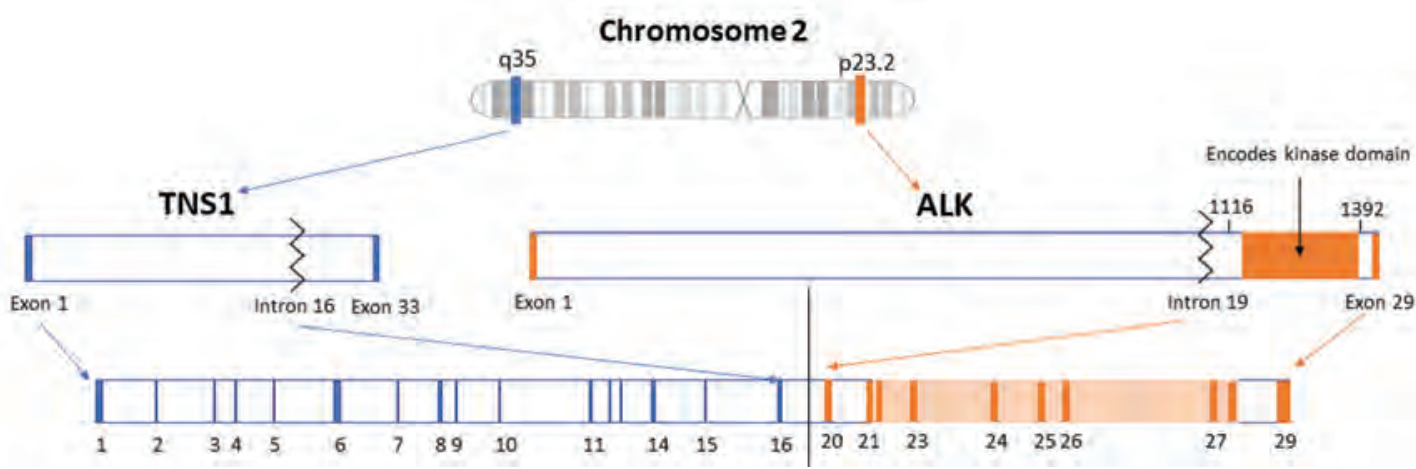

Figure 2. ALK fusion in Leiomyosarcoma. A fusion between TNS1 and ALK with breakpoints identified in intron 16 and 19 respectively was identified in the salvage colectomy specimen.

vealed radiographic partial response (Figure 1B) which has continued for 9 months at the time of submission. There were no adverse events on Brigatinib $180 \mathrm{mg}$ once daily.

\section{Discussion}

Morphologic and immunohistochemical (IHC) analyses remain important tools in classifying solid tumors, but can be complicated by inter-observer variability, overlapping IHC and morphology in rare tumors. Molecular testing is a complementary tool, capable of both identifying pathognomonic molecular alterations that aid in diagnosis while simultaneously exploring rare variants that exist across tumor types. The case presented here supports the clinical utility of CGP in advanced, recurrent, and refractory cancers.

Recent work has demonstrated that inflammatory myofibroblastic tumor (IMT) is strongly associated with kinase fusions, particularly ALK fusions $(9,10)$. Notably, IMTs often arise in the uterus, and as such can be 
misdiagnosed as leiomyosarcoma, particularly the myxoid variety (11). A case reported previously had such myxoid features and was diagnosed as a smooth muscle tumor of uncertain malignant potential, but harbored an ALK fusion and was ultimately revised to a diagnosis of uterine IMT (12). In a large series of myxoid LMS, 4 of 30 cases (13\%) were potentially under-recognized IMT based on either ALK positivity by immunohistochemistry or ALK gene rearrangements (3). The diagnosis of each is based on morphologic and immunohistochemical characteristics, but the identification of an ALK fusion/rearrangement may greatly aid in the diagnosis of difficult cases. Indeed, after re-review of morphology of available H\&E image and molecular profile, this particular tumor may be best re-classified as an IMT. At present, there are multiple reports of uterine IMT patients who harbor an ALK fusion and who have responded to an ALK inhibitor $(10,13,14)$. Molecular profiling of recurrent, metastatic uterine mesenchymal tumors may potentially aid in the diagnosis of difficult cases and enable patient enrollment in appropriate clinical trials. Owing to the rare nature of LMS and other uterine sarcomas compounded by the relative rarity of actionable alterations, randomized prospective trials are unlikely to be completed. Multi-arm basket trials matching treatments to genomic alterations, such as the ASCO TAPUR study, may be the optimal mechanism for prospective efficacy assessment as was done for this patient.

\section{Conclusion}

Identification of the TNS1-ALK fusion through CGP allowed for this tumor, originally diagnosed as LMS, to be recharacterized as an IMT. The patient also benefitted from targeted therapy, which highlights the dual role that genomic profiling can play in aiding with classifying diagnostically chal- lenging cases and enabling patients to benefit from matched targeted therapy typically within a mechanism driven clinical trial.

What Is Already Known on this Topic

Diagnosis of leiomyosarcoma based off morphologic and immunohistochemical characteristics can be challenging and a number of cases may be reclassified as IMTs as IMTs frequently harbor kinase fusions, particularly those of ALK, which can also allow for treatment with matched targeted therapy.

\section{What this Study Adds}

We report a patient with a tumor originally diagnosed as LMS that failed multiple lines of chemoradiation. CGP revealed that the tumor harbored a TNS1-ALK fusion that led to the revision of the patient's diagnosis to uterine IMT and subsequent enrollment in an ALK inhibitor trial.

Authors' Contributions: Conception and design: SMA and SJK; Acquisition, analysis, and interpretation of data: JL, AS, SMA, DIL and SJK; Drafting the article: SMA and JL; Revising it critically for important intellectual content: SMA, JL and SJK; Approved final version of the manuscript: JL, AS, SMA, DIL and SJK.

Conflict of Interest: JL, DIL, and SMA are employees of and have equity interest in Foundation Medicine, a wholly owned subsidiary of Roche Pharmaceuticals. SMA serves on the SAB of Incysus Inc., and consulting with Revolution Medicines and Takeda (non-oncology). AS declares honoraria/consultant fees from Eli Lily, Novartis, Daiichi Sankyo, and serves on the Board of Directors for Certis Oncology Solutions. SJK declares honoraria from Foundation Medicine, Inc., and consultant/advisory fees from Eli Lilly, Astellas, Boston Biomedical, and has ownership/equity in TP Therapeutics.

\section{References}

1. Gockley AA, Rauh-Hain JA, del Carmen MG. Uterine leiomyosarcoma: a review article. Int J Gynecol Cancer. 2014;24(9):1538-42.

2. Hoang HL, Ensor K, Rosen G, Leon Pachter H, Raccuia JS. Prognostic factors and survival in patients treated surgically for recurrent metastatic uterine leiomyosarcoma. Int J Surg Oncol. 2014;2014:919323.

3. Parra-Herran C, Schoolmeester JK, Yuan L, Dal Cin P, Fletcher CD, Quade BJ, et al. Myxoid Leiomyosarcoma of the Uterus: A Clinicopathologic Analysis of 30 Cases and Review of the Literature With Reappraisal of Its Distinction From Other 
Uterine Myxoid Mesenchymal Neoplasms. Am J Surg Pathol. 2016;40(3):285-301.

4. Lovly CM, Gupta A, Lipson D, Otto G, Brennan T, Chung CT, et al. Inflammatory myofibroblastic tumors harbor multiple potentially actionable kinase fusions. Cancer Discov. 2014;4(8):889-95.

5. Shaw AT, Hsu PP, Awad MM, Engelman JA. Tyrosine kinase gene rearrangements in epithelial malignancies. Nat Rev Cancer. 2013;13(11):772-87.

6. Ross JS, Ali SM, Fasan O, Block J, Pal S, Elvin JA, et al. ALK Fusions in a Wide Variety of Tumor Types Respond to Anti-ALK Targeted Therapy. Oncologist. 2017;22(12):1444-50.

7. Weinberg BA, Gowen K, Lee TK, Ou S-HI, Bristow R, Krill L, et al. Comprehensive Genomic Profiling Aids in Distinguishing Metastatic Recurrence from Second Primary Cancers. Oncologist. 2017;22(2):152-7.

8. Frampton GM, Fichtenholtz A, Otto GA, Wang K, Downing SR, He J, et al. Development and validation of a clinical cancer genomic profiling test based on massively parallel DNA sequencing. Nat Biotechnol. 2013;31(11):1023-31.

9. Antonescu CR, Suurmeijer AJ, Zhang L, Sung YS, Jungbluth AA, Travis WD, et al. Molecular characterization of inflammatory myofibroblastic tumors with frequent ALK and ROS1 gene fusions and rare novel RET rearrangement. Am J Surg Pathol. 2015;39(7):957-67.
10. Rao N, Iwenofu H, Tang B, Woyach J, Liebner DA. Inflammatory Myofibroblastic Tumor Driven by Novel NUMA1-ALK Fusion Responds to ALK Inhibition. J Natl Compr Canc Netw. 2018;16(2):115-21.

11. Fuehrer NE, Keeney GL, Ketterling RP, Knudson RA, Bell DA. ALK-1 protein expression and ALK gene rearrangements aid in the diagnosis of inflammatory myofibroblastic tumors of the female genital tract. Arch Pathol Lab Med. 2012;136(6):623-6.

12. Subbiah V, McMahon C, Patel S, Zinner R, Silva EG, Elvin JA, et al. STUMP un "stumped": antitumor response to anaplastic lymphoma kinase (ALK) inhibitor based targeted therapy in uterine inflammatory myofibroblastic tumor with myxoid features harboring DCTN1-ALK fusion. J Hematol Oncol. 2015;8:66.

13. Parker BM, Parker JV, Lymperopoulos A, Konda V. A case report: Pharmacology and resistance patterns of three generations of ALK inhibitors in metastatic inflammatory myofibroblastic sarcoma. J Oncol Pharm Pract. 2018:1078155218781944.

14. Mansfield AS, Murphy SJ, Harris FR, Robinson SI, Marks RS, Johnson SH, et al. Chromoplectic TPM3-ALK rearrangement in a patient with inflammatory myofibroblastic tumor who responded to ceritinib after progression on crizotinib. Ann Oncol. 2016;27(11):2111-7. 